\title{
Representation Theorem for Generators of BSDEs Driven by G-Brownian Motion and Its Applications
}

\author{
Kun $\mathrm{He}^{1}$ and Mingshang $\mathrm{Hu}^{2}$ \\ ${ }^{1}$ Department of Mathematics, Donghua University, 2999 North Renmin Road, Songjiang, Shanghai 201620, China \\ ${ }^{2}$ School of Mathematics, Shandong University, 27 Shanda Nanlu, Jinan 250100, China \\ Correspondence should be addressed to Kun He; hekun@dhu.edu.cn
}

Received 14 September 2013; Accepted 10 November 2013

Academic Editor: Litan Yan

Copyright (c) $2013 \mathrm{~K}$. He and M. Hu. This is an open access article distributed under the Creative Commons Attribution License, which permits unrestricted use, distribution, and reproduction in any medium, provided the original work is properly cited.

\begin{abstract}
We obtain a representation theorem for the generators of BSDEs driven by $G$-Brownian motions and then we use the representation theorem to get a converse comparison theorem for G-BSDEs and some equivalent results for nonlinear expectations generated by G-BSDEs.
\end{abstract}

\section{Introduction}

Let $(\Omega, \mathscr{F}, P)$ be a probability space, and, for fixed $T \in$ $[0,+\infty)$, let $\left(B_{t}\right)_{0 \leq t \leq T}$ be a standard Brownian motion and let $\mathscr{F}_{t}$ be the augmentation of $\sigma\left\{B_{s}, 0 \leq s \leq t\right\}$. Then Pardoux and Peng [1] introduced the backward stochastic differential equations (BSDEs) and proved the existence and uniqueness result of the BSDEs. In 1997, Peng [2] promoted $g$ expectations based on BSDEs. One of the important properties of $g$-expectations is comparison theorem or monotonicity. Chen [3] first considers a converse result of BSDEs under equal case. After that, Briand et al. [4] obtained a converse comparison theorem for BSDEs under general case. They also derived a representation theorem for the generator $g$. Following this paper, Jiang [5] discussed a more general representation theorem then, in his another paper [6], showed a more general converse comparison theorem. Here the representation theorem is an important method in solving the converse comparison problem and other problems (see Jiang [7]).

Peng [8-13] defined the G-expectations and G-Brownian motions (G-BMs) and proved the representation theorem of $G$-expectation by a set of singular probabilities, which differs from nonlinear $g$-expectations because $g$-expectations are equivalent with a group of absolutely continuous probabilities with respect to the probability measure $P$. Soner et al. [14] obtained an existence and uniqueness result of 2 BSDEs. Recently, Hu et al. [15] proved another existence and uniqueness result on BSDEs driven by $G$-Brownian motions (G-BSDEs).

An important advantage of G-BSDEs is the easiness to define the nonlinear expectations. Hu et al. in [16] gave a comparison theorem for G-BSDEs and talked about the properties of corresponding nonlinear expectations. In this paper, we consider the representation theorem for generators of G-BSDEs and then consider the converse comparison theorem of G-BSDEs and some equivalent results for nonlinear expectations generated by $G$-BSDEs. In the following, in Section 2, we review some basic concepts and results about $G$-expectations. We give the representation theorem of $G$ BSDEs in Section 3. In Section 4, we consider the applications of representation theorem of $G$-BSDEs, which contain the converse comparison theorem and some equivalent results for nonlinear expectations generated by G-BSDEs.

\section{Preliminaries}

We review some basic notions and results of $G$-expectation, the related spaces of random variables, and the backward stochastic differential equations driven by a $G$-Brownian motion. The readers may refer to $[10,13,15,17-19]$ for more details.

Definition 1. Let $\Omega$ be a given set and let $\mathscr{H}$ be a vector lattice of real valued functions defined on $\Omega$, namely, $c \in \mathscr{H}$ for each 
constant $c$ and $|X| \in \mathscr{H}$ if $X \in \mathscr{H} . \mathscr{H}$ is considered as the space of random variables. A sublinear expectation $\widehat{\mathbb{E}}$ on $\mathscr{H}$ is a functional $\widehat{\mathbb{E}}: \mathscr{H} \rightarrow \mathbb{R}$ satisfying the following properties: for all $X, Y \in \mathscr{H}$, one has

(a) monotonicity: if $X \geq Y$, then $\widehat{\mathbb{E}}[X] \geq \widehat{\mathbb{E}}[Y]$;

(b) constant preservation: $\widehat{\mathbb{E}}[c]=c$;

(c) subadditivity: $\widehat{\mathbb{E}}[X+Y] \leq \widehat{\mathbb{E}}[X]+\widehat{\mathbb{E}}[Y]$;

(d) positive homogeneity: $\widehat{\mathbb{E}}[\lambda X]=\lambda \widehat{\mathbb{E}}[X]$ for each $\lambda \geq$ $0 .(\Omega, \mathscr{H}, \widehat{\mathbb{E}})$ is called a sublinear expectation space.

Definition 2. Let $X_{1}$ and $X_{2}$ be two $n$-dimensional random vectors defined, respectively, in sublinear expectation spaces $\left(\Omega_{1}, \mathscr{H}_{1}, \widehat{\mathbb{E}}_{1}\right)$ and $\left(\Omega_{2}, \mathscr{H}_{2}, \widehat{\mathbb{E}}_{2}\right)$. They are called identically distributed, denoted by $X_{1} \stackrel{d}{=} X_{2}$, if $\widehat{\mathbb{E}}_{1}\left[\varphi\left(X_{1}\right)\right]=\widehat{\mathbb{E}}_{2}\left[\varphi\left(X_{2}\right)\right]$, for all $\varphi \in C_{b \cdot \operatorname{Lip}}\left(\mathbb{R}^{n}\right)$, where $C_{b \text {-Lip }}\left(\mathbb{R}^{n}\right)$ denotes the space of bounded and Lipschitz functions on $\mathbb{R}^{n}$.

Definition 3. In a sublinear expectation space $(\Omega, \mathscr{H}, \widehat{\mathbb{E}})$, a random vector $Y=\left(Y_{1}, \ldots, Y_{n}\right), Y_{i} \in \mathscr{H}$, is said to be independent of another random vector $X=\left(X_{1}, \ldots, X_{m}\right)$, $X_{i} \in \mathscr{H}$ under $\widehat{\mathbb{E}}[\cdot]$, denoted by $Y \perp X$, if for every test function $\varphi \in C_{b \cdot \operatorname{Lip}}\left(\mathbb{R}^{m} \times \mathbb{R}^{n}\right)$ one has $\widehat{\mathbb{E}}[\varphi(X, Y)]=$ $\widehat{\mathbb{E}}\left[\widehat{\mathbb{E}}[\varphi(x, Y)]_{x=X}\right]$.

Definition 4 (G-normal distribution). A $d$-dimensional random vector $X=\left(X_{1}, \ldots, X_{d}\right)$ in a sublinear expectation space $(\Omega, \mathscr{H}, \widehat{\mathbb{E}})$ is called $G$-normally distributed if for each $a, b \geq 0$ one has

$$
a X+b \bar{X} \stackrel{d}{=} \sqrt{a^{2}+b^{2}} X,
$$

where $\bar{X}$ is an independent copy of $X$; that is, $\bar{X} \stackrel{d}{=} X$ and $\bar{X} \perp X$. Here, the letter $G$ denotes the function

$$
G(A):=\frac{1}{2} \widehat{\mathbb{E}}[\langle A X, X\rangle]: \mathbb{S}_{d} \longrightarrow \mathbb{R}
$$

where $\mathbb{S}_{d}$ denotes the collection of $d \times d$ symmetric matrices.

Peng [13] showed that $X=\left(X_{1}, \ldots, X_{d}\right)$ is G-normally distributed if and only if for each $\varphi \in C_{b \cdot \operatorname{Lip}}\left(\mathbb{R}^{d}\right), u(t, x):=$ $\widehat{\mathbb{E}}[\varphi(x+\sqrt{t} X)],(t, x) \in[0, \infty) \times \mathbb{R}^{d}$, is the solution of the following $G$-heat equation:

$$
\partial_{t} u-G\left(D_{x}^{2} u\right)=0, \quad u(0, x)=\varphi(x) .
$$

The function $G(\cdot) \quad: \quad \mathbb{S}_{d} \rightarrow \mathbb{R}$ is a monotonic, sublinear mapping on $\mathbb{S}_{d}$ and $G(A)=(1 / 2) \widehat{\mathbb{E}}[\langle A X, X\rangle] \leq$ $(1 / 2)|A| \widehat{\mathbb{E}}\left[|X|^{2}\right]$ implies that there exists a bounded, convex, and closed subset $\Gamma \subset \mathbb{S}_{d}^{+}$such that

$$
G(A)=\frac{1}{2} \sup _{\gamma \in \Gamma} \operatorname{tr}[\gamma A],
$$

where $\mathbb{S}_{d}^{+}$denotes the collection of nonnegative elements in $\mathbb{S}_{d}$.
In this paper, we only consider nondegenerate $G$-normal distribution; that is, there exists some $\underline{\sigma}^{2}>0$ such that $G(A)-$ $G(B) \geq \underline{\sigma}^{2} \operatorname{tr}[A-B]$ for any $A \geq B$.

Definition 5. (i) Let $\Omega=C_{0}^{d}\left(\mathbb{R}^{+}\right)$denote the space of $\mathbb{R}^{d}$ valued continuous functions on $[0, \infty)$ with $\omega_{0}=0$ and let $B_{t}(\omega)=\omega_{t}$ be the canonical process. Set

$$
\begin{gathered}
L_{i p}(\Omega):=\left\{\varphi\left(B_{t_{1}}, \ldots, B_{t_{n}}\right): n \geq 1, t_{1}, \ldots, t_{n} \in[0, \infty),\right. \\
\left.\varphi \in C_{b \cdot \operatorname{Lip}}\left(\mathbb{R}^{d \times n}\right)\right\} .
\end{gathered}
$$

Let $G: \mathbb{S}_{d} \rightarrow \mathbb{R}$ be a given monotonic and sublinear function. $G$-expectation is a sublinear expectation defined by

$$
\widehat{\mathbb{E}}[X]=\widetilde{\mathbb{E}}\left[\varphi\left(\sqrt{t_{1}-t_{0}} \xi_{1}, \ldots, \sqrt{t_{m}-t_{m-1}} \xi_{m}\right)\right],
$$

for all $X=\varphi\left(B_{t_{1}}-B_{t_{0}}, B_{t_{2}}-B_{t_{1}}, \ldots, B_{t_{m}}-B_{t_{m-1}}\right)$, where $\xi_{1}, \ldots, \xi_{n}$ are identically distributed $d$-dimensional $G$ normally distributed random vectors in a sublinear expectation space $(\widetilde{\Omega}, \widetilde{\mathscr{H}}, \widetilde{\mathbb{E}})$ such that $\xi_{i+1}$ is independent of $\left(\xi_{1}, \ldots, \xi_{i}\right)$ for every $i=1, \ldots, m-1$. The corresponding canonical process $B_{t}=\left(B_{t}^{i}\right)_{i=1}^{d}$ is called a $G$-Brownian motion.

(ii) For each fixed $t \in[0, \infty)$, the conditional $G$ expectation $\widehat{\mathbb{E}}_{t}$ for $\xi=\varphi\left(B_{t_{1}}-B_{t_{0}}, B_{t_{2}}-B_{t_{1}}, \ldots, B_{t_{m}}-B_{t_{m-1}}\right) \epsilon$ $L_{i p}(\Omega)$, where without loss of generality we suppose $t_{i}=t$, is defined by

$$
\begin{aligned}
& \widehat{\mathbb{E}}_{t} {\left[\varphi\left(B_{t_{1}}-B_{t_{0}}, B_{t_{2}}-B_{t_{1}}, \ldots, B_{t_{m}}-B_{t_{m-1}}\right)\right] } \\
&=\psi\left(B_{t_{1}}-B_{t_{0}}, B_{t_{2}}-B_{t_{1}}, \ldots, B_{t_{i}}-B_{t_{i-1}}\right),
\end{aligned}
$$

where

$$
\begin{aligned}
\psi & \left(x_{1}, \ldots, x_{i}\right) \\
& =\widehat{\mathbb{E}}\left[\varphi\left(x_{1}, \ldots, x_{i}, B_{t_{i+1}}-B_{t_{i}}, \ldots, B_{t_{m}}-B_{t_{m-1}}\right)\right] .
\end{aligned}
$$

For each fixed $T>0$, we set

$$
\begin{gathered}
L_{i p}\left(\Omega_{T}\right):=\left\{\varphi\left(B_{t_{1}}, \ldots, B_{t_{n}}\right): n \geq 1, t_{1}, \ldots, t_{n} \in[0, T],\right. \\
\left.\varphi \in C_{b \cdot \operatorname{Lip}}\left(\mathbb{R}^{d \times n}\right)\right\} .
\end{gathered}
$$

For each $p \geq 1$, we denote by $L_{G}^{p}(\Omega)$ (resp., $L_{G}^{p}\left(\Omega_{T}\right)$ ) the completion of $L_{i p}(\Omega)$ (resp., $L_{i p}\left(\Omega_{T}\right)$ ) under the norm $\|\xi\|_{p, G}=\left(\widehat{\mathbb{E}}\left[|\xi|^{p}\right]\right)^{1 / p}$. It is easy to check that $L_{G}^{q}(\Omega) \subset L_{G}^{p}(\Omega)$ for $1 \leq p \leq q$ and $\widehat{\mathbb{E}}_{t}[\cdot]$ can be extended continuously to $L_{G}^{1}(\Omega)$.

For each fixed $\mathbf{a} \in \mathbb{R}^{d}, B_{t}^{\mathbf{a}}=\left\langle\mathbf{a}, B_{t}\right\rangle$ is a 1-dimensional $G_{\mathbf{a}^{-}}$ Brownian motion, where $G_{\mathbf{a}}(\alpha)=(1 / 2)\left(\sigma_{\mathbf{a a}^{T}}^{2} \alpha^{+}-\sigma_{-\mathbf{a a}^{T}}^{2} \alpha^{-}\right)$, $\sigma_{\mathbf{a a}^{T}}^{2}=2 G\left(\mathbf{a a}^{T}\right)$ and $\sigma_{-\mathbf{a a}^{T}}^{2}=-2 G\left(-\mathbf{a a}^{T}\right)$. Let $\pi_{t}^{N}=$ $\left\{t_{0}^{N}, \ldots, t_{N}^{N}\right\}, N=1,2, \ldots$, be a sequence of partitions of $[0, t]$ such that $\mu\left(\pi_{t}^{N}\right)=\max \left\{\left|t_{i+1}^{N}-t_{i}^{N}\right|: i=0, \ldots, N-1\right\} \rightarrow 0$; the quadratic variation process of $B^{\mathbf{a}}$ is defined by

$$
\left\langle B^{\mathbf{a}}\right\rangle_{t}=\lim _{\mu\left(\pi_{t}^{N}\right) \rightarrow 0} \sum_{j=0}^{N-1}\left(B_{t_{j+1}^{N}}^{\mathbf{a}}-B_{t_{j}^{N}}^{\mathbf{a}}\right)^{2} .
$$


For each fixed $\mathbf{a}, \overline{\mathbf{a}} \in \mathbb{R}^{d}$, the mutual variation process of $B^{\mathbf{a}}$ and $B^{\overline{\mathbf{a}}}$ is defined by

$$
\left\langle B^{\mathbf{a}}, B^{\overline{\mathbf{a}}}\right\rangle_{t}=\frac{1}{4}\left[\left\langle B^{\mathbf{a}+\overline{\mathbf{a}}}\right\rangle_{t}-\left\langle B^{\mathbf{a}-\overline{\mathbf{a}}}\right\rangle_{t}\right] .
$$

Definition 6. For fixed $T>0$, let $M_{G}^{0}(0, T)$ be the collection of processes in the following form: for a given partition $\left\{t_{0}, \ldots, t_{N}\right\}=\pi_{T}$ of $[0, T]$,

$$
\eta_{t}(\omega)=\sum_{j=0}^{N-1} \xi_{j} I_{\left[t_{j}, t_{j+1}\right)}(t),
$$

where $\xi_{j} \in L_{i p}\left(\Omega_{t_{j}}\right), j=0,1,2, \ldots, N-1$. For $p \geq 1$, one denotes by $H_{G}^{p}(0, T), M_{G}^{p}(0, T)$ the completion of $M_{G}^{0}(0, T)$ under the norms $\|\eta\|_{H_{G}^{p}}=\left\{\hat{\mathbb{E}}\left[\left(\int_{0}^{T}\left|\eta_{s}\right|^{2} d s\right)^{p / 2}\right]\right\}^{1 / p},\|\eta\|_{M_{G}^{p}}=$ $\left\{\widehat{\mathbb{E}}\left[\int_{0}^{T}\left|\eta_{s}\right|^{p} d s\right]\right\}^{1 / p}$, respectively.

For each $\eta \in M_{G}^{1}(0, T)$, we can define the integrals $\int_{0}^{T} \eta_{t} d t$ and $\int_{0}^{T} \eta_{t} d\left\langle B^{\mathbf{a}}, B^{\overline{\mathbf{a}}}\right\rangle_{t}$ for each a, $\overline{\mathbf{a}} \in \mathbb{R}^{d}$. For each $\eta \in$ $H_{G}^{p}\left(0, T ; \mathbb{R}^{d}\right)$ with $p \geq 1$, we can define Itô’s integral $\int_{0}^{T} \eta_{t} d B_{t}$.

Let $S_{G}^{0}(0, T)=\left\{h\left(t, B_{t_{1} \wedge t}, \ldots, B_{t_{n} \wedge t}\right): t_{1}, \ldots, t_{n} \in[0, T]\right.$, $\left.h \in C_{b, \text { Lip }}\left(\mathbb{R}^{n+1}\right)\right\}$. For $p \geq 1$ and $\eta \in S_{G}^{0}(0, T)$, set $\|\eta\|_{S_{G}^{p}}=$ $\left\{\widehat{\mathbb{E}}\left[\sup _{t \in[0, T]}\left|\eta_{t}\right|^{p}\right]\right\}^{1 / p}$. Denote by $S_{G}^{p}(0, T)$ the completion of $S_{G}^{0}(0, T)$ under the norm $\|\cdot\|_{S_{G}^{p}}$.

We consider the following type of G-BSDEs (in this paper, we always use Einstein convention):

$$
\begin{aligned}
Y_{t}= & \xi+\int_{t}^{T} f\left(s, Y_{s}, Z_{s}\right) d s+\int_{t}^{T} g_{i j}\left(s, Y_{s}, Z_{s}\right) d\left\langle B^{i}, B^{j}\right\rangle_{s} \\
& -\int_{t}^{T} Z_{s} d B_{s}-\left(K_{T}-K_{t}\right),
\end{aligned}
$$

where

$$
f(t, \omega, y, z), g_{i j}(t, \omega, y, z):[0, T] \times \Omega_{T} \times \mathbb{R} \times \mathbb{R}^{d} \longrightarrow \mathbb{R},
$$

satisfy the following properties.

(H1) There exists some $\beta>1$ such that for any

$$
y, z, f(\cdot, \cdot, y, z), g_{i j}(\cdot, \cdot, y, z) \in M_{G}^{\beta}(0, T) .
$$

(H2) There exists some $L>0$ such that

$$
\begin{aligned}
& \left|f(t, y, z)-f\left(t, y^{\prime}, z^{\prime}\right)\right|+\sum_{i, j=1}^{d}\left|g_{i j}(t, y, z)-g_{i j}\left(t, y^{\prime}, z^{\prime}\right)\right| \\
& \quad \leq L\left(\left|y-y^{\prime}\right|+\left|z-z^{\prime}\right|\right) .
\end{aligned}
$$

For simplicity, we denote by $\mathfrak{S}_{G}^{\alpha}(0, T)$ the collection of processes $(Y, Z, K)$ such that $Y \in S_{G}^{\alpha}(0, T), Z \in H_{G}^{\alpha}\left(0, T ; \mathbb{R}^{d}\right)$, $K$ is a decreasing $G$-martingale with $K_{0}=0$ and $K_{T} \in$ $L_{G}^{\alpha}\left(\Omega_{T}\right)$.
Definition 7. Let $\xi \in L_{G}^{\beta}\left(\Omega_{T}\right)$ and $f$ and $g_{i j}$ satisfy (H1) and (H2) for some $\beta>1$. A triplet of processes $(Y, Z, K)$ is called a solution of (13) if for some $1<\alpha \leq \beta$ the following properties hold:

(a) $(Y, Z, K) \in \mathbb{S}_{G}^{\alpha}(0, T)$;

(b) $Y_{t}=\xi+\int_{t}^{T} f\left(s, Y_{s}, Z_{s}\right) d s+\int_{t}^{T} g_{i j}\left(s, Y_{s}, Z_{s}\right) d\left\langle B^{i}, B^{j}\right\rangle_{s}-$ $\int_{t}^{T} Z_{s} d B_{s}-\left(K_{T}-K_{t}\right)$.

Theorem 8 (see [15]). Assume that $\xi \in L_{G}^{\beta}\left(\Omega_{T}\right)$ and $f$ and $g_{i j}$ satisfy (H1) and (H2) for some $\beta>1$. Then, (13) has a unique solution $(Y, Z, K)$. Moreover, for any $1<\alpha<\beta$, one has $Y \in$ $S_{G}^{\alpha}(0, T), Z \in H_{G}^{\alpha}\left(0, T ; \mathbb{R}^{d}\right)$, and $K_{T} \in L_{G}^{\alpha}\left(\Omega_{T}\right)$.

We have the following estimates.

Proposition 9 (see [15]). Let $\xi \in L_{G}^{\beta}\left(\Omega_{T}\right)$ and $f, g_{i j}$ satisfy (H1) and (H2) for some $\beta>1$. Assume that $(Y, Z, K) \in$ $\mathbb{S}_{G}^{\alpha}(0, T)$ for some $1<\alpha<\beta$ is a solution of (13). Then, there exists a constant $C_{\alpha}>0$ depending on $\alpha, T, G, L$ such that

$$
\begin{aligned}
& \left|Y_{t}\right|^{\alpha} \leq C_{\alpha} \widehat{\mathbb{E}}_{t}\left[|\xi|^{\alpha}+\left(\int_{t}^{T}\left|h_{s}^{0}\right| d s\right)^{\alpha}\right], \\
& \widehat{\mathbb{E}}\left[\left(\int_{0}^{T}\left|Z_{s}\right|^{2} d s\right)^{\alpha / 2}\right] \\
& \leq C_{\alpha}\left\{\widehat{\mathbb{E}}\left[\sup _{t \in[0, T]}\left|Y_{t}\right|^{\alpha}\right]+\left(\widehat{\mathbb{E}}\left[\sup _{t \in[0, T]}\left|Y_{t}\right|^{\alpha}\right]\right)^{1 / 2}\right. \\
& \left.\times\left(\widehat{\mathbb{E}}\left[\left(\int_{0}^{T} h_{s}^{0} d s\right)^{\alpha}\right]\right)^{1 / 2}\right\}, \\
& \widehat{\mathbb{E}}\left[\left|K_{t}\right|^{\alpha}\right] \leq C_{\alpha}\left\{\widehat{\mathbb{E}}\left[\sup _{t \in[0, T]}\left|Y_{t}\right|^{\alpha}\right]+\widehat{\mathbb{E}}\left[\left(\int_{0}^{T} h_{s}^{0} d s\right)^{\alpha}\right]\right\},
\end{aligned}
$$

where $h_{s}^{0}=|f(s, 0,0)|+\sum_{i, j=1}^{d}\left|g_{i j}(s, 0,0)\right|$.

Proposition 10 (see $[15,20]$ ). Let $\alpha \geq 1$ and $\delta>0$ be fixed. Then, there exists a constant $C$ depending on $\alpha$ and $\delta$ such that

$$
\begin{array}{r}
\widehat{\mathbb{E}}\left[\sup _{t \in[0, T]} \widehat{\mathbb{E}}_{t}\left[|\xi|^{\alpha}\right]\right] \\
\leq C\left\{\left(\widehat{\mathbb{E}}\left[|\xi|^{\alpha+\delta}\right]\right)^{\alpha /(\alpha+\delta)}+\widehat{\mathbb{E}}\left[|\xi|^{\alpha+\delta}\right]\right\}, \\
\forall \xi \in L_{G}^{\alpha+\delta}\left(\Omega_{T}\right) .
\end{array}
$$


Theorem 11 (see [16]). Let $\left(Y^{l}, Z^{l}, K^{l}\right), l=1,2$, be the solutions of the following G-BSDEs:

$$
\begin{aligned}
Y_{t}^{l}= & \xi+\int_{t}^{T} f\left(s, Y_{s}^{l}, Z_{s}^{l}\right) d s+\int_{t}^{T} g_{i j}\left(s, Y_{s}^{l}, Z_{s}^{l}\right) d\left\langle B^{i}, B^{j}\right\rangle_{s} \\
& +V_{T}^{l}-V_{t}^{l}-\int_{t}^{T} Z_{s}^{l} d B_{s}-\left(K_{T}^{l}-K_{t}^{l}\right),
\end{aligned}
$$

where $\xi \in L_{G}^{\beta}\left(\Omega_{T}\right)$, $f$ and $g_{i j}$ satisfy (H1) and (H2) for some $\beta>1$ and $\left(V_{t}^{l}\right)_{t \leq T}$ are RCLL processes in $M_{G}^{\beta}(0, T)$ such that $\widehat{\mathbb{E}}\left[\sup _{t \in[0, T]}\left|V_{t}^{l}\right|^{\beta}\right]<\infty$. If $V_{t}^{1}-V_{t}^{2}$ is an increasing process, then $Y_{t}^{1} \geq Y_{t}^{2}$ for $t \in[0, T]$.

In this paper, we also need the following assumptions for G-BSDE (13).

(H3) For each fixed $(\omega, y, z) \in \Omega_{T} \times \mathbb{R} \times \mathbb{R}^{d}, t \rightarrow$ $f(t, \omega, y, z)$ and $t \rightarrow g_{i j}(t, \omega, y, z)$ are continuous.

(H4) For each fixed $(t, y, z) \in[0, T) \times \mathbb{R} \times \mathbb{R}^{d}, f(t, y, z)$, $g_{i j}(t, y, z) \in L_{G}^{\beta}\left(\Omega_{t}\right)$, and

$$
\begin{aligned}
\lim _{\varepsilon \rightarrow 0+\frac{1}{\varepsilon}} \frac{1}{\mathbb{E}}\left[\int_{t}^{t+\varepsilon}(\right. & |f(u, y, z)-f(t, y, z)|^{\beta} \\
& \left.\left.+\sum_{i, j=1}^{d}\left|g_{i j}(u, y, z)-g_{i j}(t, y, z)\right|^{\beta}\right) d u\right]=0 .
\end{aligned}
$$

(H5) For each $(t, \omega, y) \in[0, T] \times \Omega_{T} \times \mathbb{R}, f(t, \omega, y, 0)=$ $g_{i j}(t, \omega, y, 0)=0$.

Assume that $\xi \in L_{G}^{\beta}\left(\Omega_{T}\right) ; f$ and $g_{i j}$ satisfy (H1), (H2), and (H5) for some $\beta>1$. Let $\left(Y^{T, \xi}, Z^{T, \xi}, K^{T, \xi}\right)$ be the solution of G-BSDE (13) corresponding to $\xi$, $f$, and $g_{i j}$ on $[0, T]$. It is easy to check that $Y^{T, \xi}=Y^{T^{\prime}, \xi}$ on $[0, T]$ for $T^{\prime}>T$. Following [16], we can define consistent nonlinear expectation

$$
\widetilde{\mathbb{E}}_{t}[\xi]=Y_{t}^{T, \xi} \quad \text { for } t \in[0, T]
$$

and set $\widetilde{\mathbb{E}}[\xi]=\widetilde{\mathbb{E}}_{0}[\xi]=Y_{0}^{T, \xi}$.

\section{Representation Theorem of Generators of $G$-BSDEs}

We consider the following type of G-FBSDEs:

$$
\begin{aligned}
X_{s}^{t, x}= & x+\int_{t}^{s} b\left(X_{u}^{t, x}\right) d u \\
& +\int_{t}^{s} h_{i j}\left(X_{u}^{t, x}\right) d\left\langle B^{i}, B^{j}\right\rangle_{u}+\int_{t}^{s} \sigma\left(X_{u}^{t, x}\right) d B_{u},
\end{aligned}
$$

$$
\begin{aligned}
{ }^{\varepsilon} Y_{s}^{t, x, y, p}= & y+\left\langle p, X_{t+\varepsilon}^{t, x}-x\right\rangle \\
& +\int_{s}^{t+\varepsilon} f\left(u,{ }^{\varepsilon} Y_{u}^{t, x, y, p},{ }^{\varepsilon} Z_{u}^{t, x, y, p}\right) d u \\
& +\int_{s}^{t+\varepsilon} g_{i j}\left(u,{ }^{\varepsilon} Y_{u}^{t, x, y, p},{ }^{\varepsilon} Z_{u}^{t, x, y, p}\right) d\left\langle B^{i}, B^{j}\right\rangle_{u} \\
& -\int_{s}^{t+\varepsilon}{ }^{t} Z_{u}^{t, x, y, p} d B_{u}-\left({ }^{\varepsilon} K_{t+\varepsilon}^{t, x, y, p}-{ }^{\varepsilon} K_{s}^{t, x, y, p}\right),
\end{aligned}
$$

where $h_{i j}=h_{j i}$ and $g_{i j}=g_{j i}, 1 \leq i, j \leq d$.

We now give the main result in this section.

Theorem 12. Let $b: \mathbb{R}^{n} \rightarrow \mathbb{R}^{n}, h_{i j}: \mathbb{R}^{n} \rightarrow \mathbb{R}^{n}$, and $\sigma:$ $\mathbb{R}^{n} \rightarrow \mathbb{R}^{n \times d}$ be Lipschitz functions and let $f$ and $g_{i j}$ satisfy (H1), (H2), (H3), and (H4) for some $\beta>1$. Then, for each $(t, x, y, p) \in[0, T) \times \mathbb{R}^{n} \times \mathbb{R} \times \mathbb{R}^{n}$ and $\alpha \in(1, \beta)$, one has

$$
\begin{aligned}
L_{G}^{\alpha}- & \lim _{\varepsilon \rightarrow 0+} \frac{1}{\varepsilon}\left\{{ }^{\varepsilon} Y_{t}^{t, x, y, p}-y\right\} \\
= & f\left(t, y, \sigma^{T}(x) p\right)+\langle p, b(x)\rangle \\
& +2 G\left(\left(g_{i j}\left(t, y, \sigma^{T}(x) p\right)+\left\langle p, h_{i j}(x)\right\rangle\right)_{i, j=1}^{d}\right) .
\end{aligned}
$$

Proof. For each fixed $(t, x, y, p) \in[0, T) \times \mathbb{R}^{n} \times \mathbb{R} \times \mathbb{R}^{n}$, we write $\left(Y^{\varepsilon}, Z^{\varepsilon}, K^{\varepsilon}\right)$ instead of $\left({ }^{\varepsilon} Y^{t, x, y, p},{ }^{\varepsilon} Z^{t, x, y, p},{ }^{\varepsilon} K^{t, x, y, p}\right)$ for simplicity. We have $\widehat{\mathbb{E}}\left[\left|X_{t+\varepsilon}^{t, x}\right|^{\gamma}\right]<\infty$ for each $\gamma \geq 1$ (see $[16,19])$. Thus, by Theorem $8, G$-BSDE (22) has a unique solution $\left(Y^{\varepsilon}, Z^{\varepsilon}, K^{\varepsilon}\right)$ and $Y_{t}^{\varepsilon} \in L_{G}^{\alpha}\left(\Omega_{t}\right)$. We set, for $s \in[t, t+\varepsilon]$,

$$
\begin{aligned}
& \widetilde{Y}_{s}^{\varepsilon}=Y_{s}^{\varepsilon}-\left(y+\left\langle p, X_{s}^{t, x}-x\right\rangle\right), \\
& \widetilde{Z}_{s}^{\varepsilon}=Z_{s}^{\varepsilon}-\sigma^{T}\left(X_{s}^{t, x}\right) p, \quad \widetilde{K}_{s}^{\varepsilon}=K_{s}^{\varepsilon} .
\end{aligned}
$$

Applying Itô's formula to $\widetilde{Y}_{s}^{\varepsilon}$ on $[t, t+\varepsilon]$, it is easy to verify that $\left(\widetilde{Y}^{\varepsilon}, \widetilde{Z}^{\varepsilon}, \widetilde{K}^{\varepsilon}\right)$ solves the following $G$-BSDE:

$$
\begin{aligned}
& \widetilde{Y}_{s}^{\varepsilon}= \int_{s}^{t+\varepsilon} f\left(u, \widetilde{Y}_{u}^{\varepsilon}+y+\left\langle p, X_{u}^{t, x}-x\right\rangle,\right. \\
&\left.\widetilde{Z}_{u}^{\varepsilon}+\sigma^{T}\left(X_{u}^{t, x}\right) p\right) d u \\
&+\int_{s}^{t+\varepsilon}\left\langle p, b\left(X_{u}^{t, x}\right)\right\rangle d u \\
&+\int_{s}^{t+\varepsilon} g_{i j}\left(u, \widetilde{Y}_{u}^{\varepsilon}+y+\left\langle p, X_{u}^{t, x}-x\right\rangle,\right. \\
&\left.\quad \widetilde{Z}_{u}^{\varepsilon}+\sigma^{T}\left(X_{u}^{t, x}\right) p\right) d\left\langle B^{i}, B^{j}\right\rangle_{u} \\
&+\int_{s}^{t+\varepsilon}\left\langle p, h_{i j}\left(X_{u}^{t, x}\right)\right\rangle d\left\langle B^{i}, B^{j}\right\rangle_{u} \\
&-\int_{s}^{t+\varepsilon} \widetilde{Z}_{u}^{\varepsilon} d B_{u}-\left(\widetilde{K}_{t+\varepsilon}^{\varepsilon}-\widetilde{K}_{s}^{\varepsilon}\right) .
\end{aligned}
$$


From Proposition 9,

$$
\begin{aligned}
& \begin{aligned}
\left|\widetilde{Y}_{s}^{\varepsilon}\right|^{\alpha} \leq C_{\alpha} \widehat{\mathbb{E}}_{s}\left[\left(\int_{s}^{t+\varepsilon}\right.\right. & \left(\left|f\left(u, y+\left\langle p, X_{u}^{t, x}-x\right\rangle, \sigma^{T}\left(X_{u}^{t, x}\right) p\right)\right|\right. \\
& +\left|\left\langle p, b\left(X_{u}^{t, x}\right)\right\rangle\right| \\
& +\sum_{i, j=1}^{d}\left|g_{i j}\left(u, y+\left\langle p, X_{u}^{t, x}-x\right\rangle, \sigma^{T}\left(X_{u}^{t, x}\right) p\right)\right| \\
& \left.\left.\left.+\left|\left\langle p, h_{i j}\left(X_{u}^{t, x}\right)\right\rangle\right|\right) d u\right)^{\alpha}\right],
\end{aligned} \\
& \begin{aligned}
\widehat{\mathbb{E}}\left[\left(\int_{t}^{t+\varepsilon}\left|\widetilde{Z}_{u}^{\varepsilon}\right|^{2} d u\right)^{\alpha / 2}\right] \\
\leq C_{\alpha}\left\{\widehat { \mathbb { E } } \left[\left(\int _ { t } ^ { t + \varepsilon } \left(\left|f\left(u, y+\left\langle p, X_{u}^{t, x}-x\right\rangle, \sigma^{T}\left(X_{u}^{t, x}\right) p\right)\right|\right.\right.\right.\right. \\
+\left|\left\langle p, b\left(X_{u}^{t, x}\right)\right\rangle\right|+\left|\left\langle p, h_{i j}\left(X_{u}^{t, x}\right)\right\rangle\right|
\end{aligned} \\
& +\sum_{i, j=1}^{d} \mid g_{i j}\left(u, y+\left\langle p, X_{u}^{t, x}-x\right\rangle,\right. \\
& \left.+\widehat{\mathbb{E}}\left[\sup _{s \in[t, t+\varepsilon]}\left|\widetilde{Y}_{s}^{\varepsilon}\right|^{\alpha}\right]\right\},
\end{aligned}
$$

hold for some constant $C_{\alpha}>0$, only depending on $\alpha, T, G$, and $L$. By Proposition 10 and the Lipschitz assumption, we obtain

$$
\begin{aligned}
& \widehat{\mathbb{E}}\left[\sup _{s \in[t, t+\varepsilon]}\left|\widetilde{Y}_{s}^{\varepsilon}\right|^{\alpha}+\left(\int_{t}^{t+\varepsilon}\left|\widetilde{Z}_{u}^{\varepsilon}\right|^{2} d u\right)^{\alpha / 2}\right] \\
& \leq C_{1} \varepsilon^{\alpha} \widehat{\mathbb{E}}\left[1+\left(\frac { 1 } { \varepsilon } \int _ { t } ^ { t + \varepsilon } \left(|f(u, 0,0)|^{\beta}\right.\right.\right. \\
& \left.\left.+\sum_{i, j=1}^{d}\left|g_{i j}(u, 0,0)\right|^{\beta}\right) d u\right)^{\alpha / \beta} \\
& \left.+\sup _{s \in[t, t+\varepsilon]}\left|X_{s}^{t, x}\right|^{\beta}\right]
\end{aligned}
$$

where $C_{1}$ is a constant depending on $x, y, p, \alpha, \beta, T, G$, and $L$. Noting that $\widehat{\mathbb{E}}\left[\sup _{s \in[t, t+\varepsilon]}\left|X_{s}^{t, x}\right|^{\beta}\right] \leq C_{2}\left(1+|x|^{\beta}\right)$ (see $\left.[16,19]\right)$, where $C_{2}$ depends on $T$ and $L$, and the following inequality holds:

$$
\begin{aligned}
\int_{\mathrm{t}}^{t+\varepsilon}\left(|f(u, 0,0)|^{\beta}+\sum_{i, j=1}^{d}\left|g_{i j}(u, 0,0)\right|^{\beta}\right) d u \\
\leq 2^{\beta-1}\left\{\varepsilon\left(|f(t, 0,0)|^{\beta}+\sum_{i, j=1}^{d}\left|g_{i j}(t, 0,0)\right|^{\beta}\right)\right. \\
+\int_{t}^{t+\varepsilon}\left(|f(u, 0,0)-f(t, 0,0)|^{\beta}\right. \\
\left.\left.+\sum_{i, j=1}^{d}\left|g_{i j}(u, 0,0)-g_{i j}(t, 0,0)\right|^{\beta}\right) d u\right\} .
\end{aligned}
$$

Together with assumption (H4), we get

$$
\widehat{\mathbb{E}}\left[\sup _{s \in[t, t+\varepsilon]}\left|\widetilde{Y}_{s}^{\varepsilon}\right|^{\alpha}+\left(\int_{t}^{t+\varepsilon}\left|\widetilde{Z}_{u}^{\varepsilon}\right|^{2} d u\right)^{\alpha / 2}\right] \leq C_{3} \varepsilon^{\alpha}
$$

where $C_{3}$ depends on $x, y, p, \alpha, \beta, T, G$, and $L$. Now, we prove (23). Let us consider

$$
\begin{aligned}
\frac{1}{\varepsilon}\left\{Y_{t}^{\varepsilon}-y\right\} & =\frac{1}{\varepsilon} \widetilde{Y}_{t}^{\varepsilon}=\frac{1}{\varepsilon} \widehat{\mathbb{E}}_{t}\left[\widetilde{Y}_{t}^{\varepsilon}+\widetilde{K}_{t+\varepsilon}^{\varepsilon}-\widetilde{K}_{t}^{\varepsilon}\right] \\
=\frac{1}{\varepsilon} \widehat{\mathbb{E}}_{t}\left[\int_{t}^{t+\varepsilon} f\left(u, y+\left\langle p, X_{u}^{t, x}-x\right\rangle, \sigma^{T}\left(X_{u}^{t, x}\right) p\right) d u\right. & \\
+ & \int_{t}^{t+\varepsilon}\left\langle p, b\left(X_{u}^{t, x}\right)\right\rangle d u \\
& +\int_{t}^{t+\varepsilon} g_{i j}\left(u, y+\left\langle p, X_{u}^{t, x}-x\right\rangle, \sigma^{T}\left(X_{u}^{t, x}\right) p\right) \\
& \times d\left\langle B^{i}, B^{j}\right\rangle{ }_{u} \\
& \left.+\int_{t}^{t+\varepsilon}\left\langle p, h_{i j}\left(X_{u}^{t, x}\right)\right\rangle d\left\langle B^{i}, B^{j}\right\rangle_{u}\right]+L_{\varepsilon},
\end{aligned}
$$

where

$$
\begin{aligned}
& L_{\varepsilon}=\frac{1}{\varepsilon}\left\{\widehat { \mathbb { E } } _ { t } \left[\int _ { t } ^ { t + \varepsilon } f \left(u, \widetilde{Y}_{u}^{\varepsilon}+y+\left\langle p, X_{u}^{t, x}-x\right\rangle,\right.\right.\right. \\
& \left.\widetilde{Z}_{u}^{\varepsilon}+\sigma^{T}\left(X_{u}^{t, x}\right) p\right) d u \\
& +\int_{t}^{t+\varepsilon}\left\langle p, b\left(X_{u}^{t, x}\right)\right\rangle d u \\
& +\int_{t}^{t+\varepsilon} g_{i j}\left(u, \tilde{Y}_{u}^{\varepsilon}+y+\left\langle p, X_{u}^{t, x}-x\right\rangle,\right. \\
& \left.\widetilde{Z}_{u}^{\varepsilon}+\sigma^{T}\left(X_{u}^{t, x}\right) p\right) d\left\langle B^{i}, B^{j}\right\rangle_{u} \\
& \left.+\int_{t}^{t+\varepsilon}\left\langle p, h_{i j}\left(X_{u}^{t, x}\right)\right\rangle d\left\langle B^{i}, B^{j}\right\rangle_{u}\right] \\
& -\widehat{\mathbb{E}}_{t}\left[\int_{t}^{t+\varepsilon} f\left(u, y+\left\langle p, X_{u}^{t, x}-x\right\rangle, \sigma^{T}\left(X_{u}^{t, x}\right) p\right) d u\right. \\
& +\int_{t}^{t+\varepsilon}\left\langle p, b\left(X_{u}^{t, x}\right)\right\rangle d u \\
& +\int_{t}^{t+\varepsilon} g_{i j}\left(u, y+\left\langle p, X_{u}^{t, x}-x\right\rangle, \sigma^{T}\left(X_{u}^{t, x}\right) p\right) \\
& \times d\left\langle B^{i}, B^{j}\right\rangle_{u} \\
& \left.\left.+\int_{t}^{t+\varepsilon}\left\langle p, h_{i j}\left(X_{u}^{t, x}\right)\right\rangle d\left\langle B^{i}, B^{j}\right\rangle_{u}\right]\right\}
\end{aligned}
$$


It is easy to check that $\left|L_{\varepsilon}\right| \leq\left(C_{4} / \varepsilon\right) \widehat{\mathbb{E}}_{t}\left[\int_{t}^{t+\varepsilon}\left(\left|\widetilde{Y}_{u}^{\varepsilon}\right|+\left|\widetilde{Z}_{u}^{\varepsilon}\right|\right) d u\right]$, where $C_{4}$ depends on $G, L$, and $T$. Thus, by (29), we get

$$
\begin{aligned}
& \widehat{\mathbb{E}}\left[\left|L_{\varepsilon}\right|^{\alpha}\right] \\
& \leq \frac{C_{4}^{\alpha}}{\varepsilon^{\alpha}} \widehat{\mathbb{E}}\left[\left(\int_{t}^{t+\varepsilon}\left(\left|\widetilde{Y}_{u}^{\varepsilon}\right|+\left|\widetilde{Z}_{u}^{\varepsilon}\right|\right) d u\right)^{\alpha}\right] \\
& \leq \frac{2^{\alpha-1} C_{4}^{\alpha} \widehat{\mathbb{E}}\left[\left(\int_{t}^{t+\varepsilon}\left|\widetilde{Y}_{u}^{\varepsilon}\right| d u\right)^{\alpha}+\left(\int_{t}^{t+\varepsilon}\left|\widetilde{Z}_{u}^{\varepsilon}\right| d u\right)^{\alpha}\right]}{\leq 2^{\alpha-1} C_{4}^{\alpha}\left\{\widehat{\mathbb{E}}\left[\sup _{s \in[t, t+\varepsilon]}\left|\widetilde{Y}_{s}^{\varepsilon}\right|^{\alpha}\right]+\varepsilon^{-\alpha / 2} \widehat{\mathbb{E}}\left[\left(\int_{t}^{t+\varepsilon}\left|\widetilde{Z}_{u}^{\varepsilon}\right|^{2} d u\right)^{\alpha / 2}\right]\right\}} \\
& \leq 2^{\alpha-1} C_{4}^{\alpha} C_{3}\left(\varepsilon^{\alpha}+\varepsilon^{\alpha / 2}\right),
\end{aligned}
$$

which implies $L_{G}^{\alpha}-\lim _{\varepsilon \rightarrow 0+} L_{\varepsilon}=0$. We set

$$
\begin{gathered}
M_{\varepsilon}=\frac{1}{\varepsilon}\left\{\widehat { \mathbb { E } } _ { t } \left[\int_{t}^{t+\varepsilon} f\left(u, y+\left\langle p, X_{u}^{t, x}-x\right\rangle, \sigma^{T}\left(X_{u}^{t, x}\right) p\right) d u\right.\right. \\
+\quad \int_{t}^{t+\varepsilon}\left\langle p, b\left(X_{u}^{t, x}\right)\right\rangle d u \\
+\int_{t}^{t+\varepsilon} g_{i j}\left(u, y+\left\langle p, X_{u}^{t, x}-x\right\rangle, \sigma^{T}\left(X_{u}^{t, x}\right) p\right) \\
\quad \times d\left\langle B^{i}, B^{j}\right\rangle_{u} \\
\left.+\int_{t}^{t+\varepsilon}\left\langle p, h_{i j}\left(X_{u}^{t, x}\right)\right\rangle d\left\langle B^{i}, B^{j}\right\rangle_{u}\right] \\
-\widehat{\mathbb{E}}_{t}\left[\int_{t}^{t+\varepsilon} f\left(u, y, \sigma^{T}(x) p\right) d u+\langle p, b(x)\rangle \varepsilon\right. \\
+\int_{t}^{t+\varepsilon} g_{i j}\left(u, y, \sigma^{T}(x) p\right) d\left\langle B^{i}, B^{j}\right\rangle_{u} \\
\left.\left.+\int_{t}^{t+\varepsilon}\left\langle p, h_{i j}(x)\right\rangle d\left\langle B^{i}, B^{j}\right\rangle_{u}\right]\right\} .
\end{gathered}
$$

By the Lipschitz condition, we can get $\left|M_{\varepsilon}\right| \leq$ $\left(C_{5} / \varepsilon\right) \widehat{\mathbb{E}}_{t}\left[\int_{t}^{t+\varepsilon}\left|X_{u}^{t, x}-x\right| d u\right]$, where $C_{5}$ depends on $p, G, L$, and $T$. Noting that $\widehat{\mathbb{E}}\left[\sup _{s \in[t, t+\varepsilon]}\left|X_{s}^{t, x}-x\right|^{\alpha}\right] \leq C_{6}\left(1+|x|^{\alpha}\right) \varepsilon^{\alpha / 2}$ (see $[16,19]$ ), where $C_{6}$ depends on $L, G$, and $\alpha$, we obtain

$$
\begin{aligned}
\widehat{\mathbb{E}}\left[\left|M_{\varepsilon}\right|^{\alpha}\right] & \leq C_{5}^{\alpha} \widehat{\mathbb{E}}\left[\sup _{s \in[t, t+\varepsilon]}\left|X_{s}^{t, x}-x\right|^{\alpha}\right] \\
& \leq C_{5}^{\alpha} C_{6}\left(1+|x|^{\alpha}\right) \varepsilon^{\alpha / 2},
\end{aligned}
$$

which implies $L_{G}^{\alpha}-\lim _{\varepsilon \rightarrow 0+} M_{\varepsilon}=0$. Now, we set

$$
\begin{array}{r}
N_{\varepsilon}=\frac{1}{\varepsilon}\left\{\widehat { \mathbb { E } } _ { t } \left[\int_{t}^{t+\varepsilon} f\left(u, y, \sigma^{T}(x) p\right) d u+\langle p, b(x)\rangle_{\varepsilon}\right.\right. \\
\quad+\int_{t}^{t+\varepsilon} g_{i j}\left(u, y, \sigma^{T}(x) p\right) d\left\langle B^{i}, B^{j}\right\rangle_{u} \\
\left.\quad+\int_{t}^{t+\varepsilon}\left\langle p, h_{i j}(x)\right\rangle d\left\langle B^{i}, B^{j}\right\rangle_{u}\right] \\
-\widehat{\mathbb{E}}_{t}\left[\int_{t}^{t+\varepsilon} f\left(t, y, \sigma^{T}(x) p\right) d u+\langle p, b(x)\rangle \varepsilon\right. \\
+\int_{t}^{t+\varepsilon} g_{i j}\left(t, y, \sigma^{T}(x) p\right) d\left\langle B^{i}, B^{j}\right\rangle_{u} \\
\left.\left.+\int_{t}^{t+\varepsilon}\left\langle p, h_{i j}(x)\right\rangle d\left\langle B^{i}, B^{j}\right\rangle_{u}\right]\right\}
\end{array}
$$

It is easy to deduce that $\left|N_{\varepsilon}\right| \leq\left(C_{7} / \varepsilon\right) \widehat{\mathbb{E}}_{t}\left[\int_{t}^{t+\varepsilon}(\mid f(u, y\right.$, $\left.\sigma^{T}(x) p\right)-f\left(t, y, \sigma^{T}(x) p\right)\left|+\sum_{i, j=1}^{d}\right| g_{i j}\left(u, y, \sigma^{T}(x) p\right)-g_{i j}(t, y$, $\left.\left.\sigma^{T}(x) p\right) \mid\right) d u$ ], where $C_{7}$ depends on $G$. Then,

$$
\begin{aligned}
& \widehat{\mathbb{E}}\left[\left|N_{\varepsilon}\right|^{\alpha}\right] \\
& \leq C_{7}^{\alpha} \frac{1}{\varepsilon} \widehat{\mathbb{E}}\left[\int _ { t } ^ { t + \varepsilon } \left(\left|f\left(u, y, \sigma^{T}(x) p\right)-f\left(t, y, \sigma^{T}(x) p\right)\right|\right.\right. \\
& +\sum_{i, j=1}^{d} \mid g_{i j}\left(u, y, \sigma^{T}(x) p\right) \\
& \leq C_{7}^{\alpha}\left(\frac { 1 } { \varepsilon } \widehat { \mathbb { E } } \left[\int _ { t } ^ { t + \varepsilon } \left(\mid f\left(u, y, \sigma^{T}(x) p\right)\right.\right.\right. \\
& -f\left(t, y, \sigma^{T}(x) p\right) \mid \\
& \left.\left.+\sum_{i, j=1}^{d}\left|g_{i j}\left(u, y, \sigma^{T}(x) p\right)\right|\right)^{\alpha} d u\right]
\end{aligned}
$$

$$
\left.\left.\left.-g_{i j}\left(t, y, \sigma^{T}(x) p\right) \mid\right)^{\beta} d u\right]\right)^{\alpha / \beta}
$$

Take limit on both sides of the above inequality and use assumption (H4); then, we have

$$
L_{G}^{\alpha}-\lim _{\varepsilon \rightarrow 0+} N_{\varepsilon}=0
$$


On the other hand,

$$
\begin{aligned}
& \widehat{\mathbb{E}}_{t}\left[\int_{t}^{t+\varepsilon} f\left(t, y, \sigma^{T}(x) p\right) d u+\langle p, b(x)\rangle \varepsilon\right. \\
& \quad+\int_{t}^{t+\varepsilon} g_{i j}\left(t, y, \sigma^{T}(x) p\right) d\left\langle B^{i}, B^{j}\right\rangle_{u} \\
& \left.\quad+\int_{t}^{t+\varepsilon}\left\langle p, h_{i j}(x)\right\rangle d\left\langle B^{i}, B^{j}\right\rangle_{u}\right] \\
& =f\left(t, y, \sigma^{T}(x) p\right) \varepsilon+\langle p, b(x)\rangle \varepsilon \\
& \quad+\widehat{\mathbb{E}}_{t}\left[\left(g_{i j}\left(t, y, \sigma^{T}(x) p\right)+\left\langle p, h_{i j}(x)\right\rangle\right)\right. \\
& \left.\quad \times\left(\left\langle B^{i}, B^{j}\right\rangle_{t+\varepsilon}-\left\langle B^{i}, B^{j}\right\rangle_{t}\right)\right] \\
& =\left(f\left(t, y, \sigma^{T}(x) p\right)+\langle p, b(x)\rangle\right. \\
& \left.\quad+2 G\left(\left(g_{i j}\left(t, y, \sigma^{T}(x) p\right)+\left\langle p, h_{i j}(x)\right\rangle\right)_{i, j=1}^{d}\right)\right) \varepsilon .
\end{aligned}
$$

Then, we have

$$
\begin{aligned}
L_{G}^{\alpha}- & \lim _{\varepsilon \rightarrow 0+} \frac{1}{\varepsilon}\left\{Y_{t}^{\varepsilon}-y\right\} \\
= & f\left(t, y, \sigma^{T}(x) p\right)+\langle p, b(x)\rangle \\
& +2 G\left(\left(g_{i j}\left(t, y, \sigma^{T}(x) p\right)+\left\langle p, h_{i j}(x)\right\rangle\right)_{i, j=1}^{d}\right) .
\end{aligned}
$$

The proof is complete.

\section{Some Applications}

4.1. Converse Comparison Theorem for G-BSDEs. We consider the following $G$-BSDEs:

$$
\begin{aligned}
Y_{t}^{l, \xi}= & \xi+\int_{t}^{T} f^{l}\left(s, Y_{s}^{l, \xi}, Z_{s}^{l, \xi}\right) d s \\
& +\int_{t}^{T} g_{i j}^{l}\left(s, Y_{s}^{l, \xi}, Z_{s}^{l, \xi}\right) d\left\langle B^{i}, B^{j}\right\rangle_{s} \\
& -\int_{t}^{T} Z_{s}^{l, \xi} d B_{s}-\left(K_{T}^{l, \xi}-K_{t}^{l, \xi}\right), \quad l=1,2,
\end{aligned}
$$

where $g_{i j}^{l}=g_{j i}^{l}$.

We first generalized the comparison theorem in [16].

Proposition 13. Let $f^{l}$ and $g_{i j}^{l}$ satisfy (H1) and (H2) for some $\beta>1, l=1,2$. If $f^{2}-f^{1}+2 G\left(\left(g_{i j}^{2}-g_{i j}^{1}\right)_{i, j=1}^{d}\right) \leq 0$, then, for each $\xi \in L_{G}^{\beta}\left(\Omega_{T}\right)$, one has $Y_{t}^{1, \xi} \geq Y_{t}^{2, \xi}$ for $t \in[0, T]$.
Proof. From the above G-BSDEs, we have

$$
\begin{aligned}
Y_{t}^{2, \xi}= & \xi+\int_{t}^{T} f^{2}\left(s, Y_{s}^{2, \xi}, Z_{s}^{2, \xi}\right) d s \\
& +\int_{t}^{T} g_{i j}^{2}\left(s, Y_{s}^{2, \xi}, Z_{s}^{2, \xi}\right) d\left\langle B^{i}, B^{j}\right\rangle_{s} \\
& -\int_{t}^{T} Z_{s}^{2, \xi} d B_{s}-\left(K_{T}^{2, \xi}-K_{t}^{2, \xi}\right) \\
= & \xi \int_{t}^{T} f^{1}\left(s, Y_{s}^{2, \xi}, Z_{s}^{2, \xi}\right) d s \\
& +\int_{t}^{T} g_{i j}^{1}\left(s, Y_{s}^{2, \xi}, Z_{s}^{2, \xi}\right) d\left\langle B^{i}, B^{j}\right\rangle_{s} \\
& +V_{T}-V_{t}-\int_{t}^{T} Z_{s}^{2, \xi} d B_{s}-\left(K_{T}^{2, \xi}-K_{t}^{2, \xi}\right),
\end{aligned}
$$

where

$$
\begin{aligned}
V_{t}= & \int_{0}^{t}\left(f^{2}-f^{1}\right)\left(s, Y_{s}^{2, \xi}, Z_{s}^{2, \xi}\right) d s \\
& +\int_{0}^{t}\left(g_{i j}^{2}-g_{i j}^{1}\right)\left(s, Y_{s}^{2, \xi}, Z_{s}^{2, \xi}\right) d\left\langle B^{i}, B^{j}\right\rangle_{s} \\
= & \int_{0}^{t}\left(f^{2}-f^{1}+2 G\left(\left(g_{i j}^{2}-g_{i j}^{1}\right)_{i, j=1}^{d}\right)\right)\left(s, Y_{s}^{2, \xi}, Z_{s}^{2, \xi}\right) d s \\
& +\int_{0}^{t}\left(g_{i j}^{2}-g_{i j}^{1}\right)\left(s, Y_{s}^{2, \xi}, Z_{s}^{2, \xi}\right) d\left\langle B^{i}, B^{j}\right\rangle_{s} \\
& -\int_{0}^{t} 2 G\left(\left(g_{i j}^{2}-g_{i j}^{1}\right)_{i, j=1}^{d}\right)\left(s, Y_{s}^{2, \xi}, Z_{s}^{2, \xi}\right) d s .
\end{aligned}
$$

By the assumption, it is easy to check that $\left(V_{t}\right)_{t \leq T}$ is a decreasing process. Thus, using Theorem 11, we obtain $Y_{t}^{1, \xi} \geq$ $Y_{t}^{2, \xi}$ for $t \in[0, T]$.

Remark 14. Suppose $d=1$ and let $f^{1}=10|z|, f^{2}=|z|$, $g^{1}=|z|$, and $g^{2}=2|z|$. It is easy to check that $f^{2}-f^{1}+$ $2 G\left(g^{2}-g^{1}\right) \leq 0$. Thus, $f^{2}-f^{1}+2 G\left(\left(g_{i j}^{2}-g_{i j}^{1}\right)_{i, j=1}^{d}\right) \leq 0$ does not imply $f^{2} \leq f^{1}$ and $\left(g_{i j}^{2}\right)_{i, j=1}^{d} \leq\left(g_{i j}^{1}\right)_{i, j=1}^{d}$.

Now, we give the converse comparison theorem.

Theorem 15. Let $f^{l}$ and $g_{i j}^{l}$ satisfy (H1), (H2), (H3), (H4), and (H5) for some $\beta>1, l=1,2$. If $Y_{t}^{1, \xi} \geq Y_{t}^{2, \xi}$ for each $t \in[0, T]$ and $\xi \in L_{G}^{\beta}\left(\Omega_{T}\right)$, then $f^{2}-f^{1}+2 G\left(\left(g_{i j}^{2}-g_{i j}^{1}\right)_{i, j=1}^{d}\right) \leq 0$ q.s..

Proof. For simplicity, we take the notation $\widetilde{\mathbb{E}}_{t}^{l}[\xi]=Y_{t}^{l, \xi}, l=$ 1,2 . For each fixed $(t, y, z) \in[0, T) \times \mathbb{R} \times \mathbb{R}^{d}$, let us consider

$$
\eta_{\varepsilon}=y+\left\langle z, h_{i j}\right\rangle\left(\left\langle B^{i}, B^{j}\right\rangle_{t+\varepsilon}-\left\langle B^{i}, B^{j}\right\rangle_{t}\right)+\left\langle z, B_{t+\varepsilon}-B_{t}\right\rangle,
$$


where $h_{i j}=h_{j i} \in \mathbb{R}^{d}$. By Theorem 12, we have, for each $\alpha \in$ $(1, \beta)$,

$$
\begin{aligned}
L_{G}^{\alpha} & -\lim _{\varepsilon \rightarrow 0+} \frac{1}{\varepsilon}\left(\widetilde{\mathbb{E}}_{t}^{l}\left[\eta_{\varepsilon}\right]-y\right) \\
& =f^{l}(t, y, z)+2 G\left(\left(g_{i j}^{l}(t, y, z)+\left\langle z, h_{i j}\right\rangle\right)_{i, j=1}^{d}\right) .
\end{aligned}
$$

Since $\widetilde{\mathbb{E}}_{t}^{1}\left[\eta_{\varepsilon}\right] \geq \widetilde{\mathbb{E}}_{t}^{2}\left[\eta_{\varepsilon}\right]$,

$$
\begin{aligned}
& f^{1}(t, y, z)+2 G\left(\left(g_{i j}^{1}(t, y, z)+\left\langle z, h_{i j}\right\rangle\right)_{i, j=1}^{d}\right) \\
& \quad \geq f^{2}(t, y, z)+2 G\left(\left(g_{i j}^{2}(t, y, z)+\left\langle z, h_{i j}\right\rangle\right)_{i, j=1}^{d}\right) \text { q.s. }
\end{aligned}
$$

Take a $h_{i j}$ such that $\left\langle z, h_{i j}\right\rangle=-g_{i j}^{1}(t, y, z)$. Therefore, $\left\{f^{2}-f^{1}+\right.$ $\left.2 G\left(\left(g_{i j}^{2}-g_{i j}^{1}\right)_{i, j=1}^{d}\right)\right\}(t, y, z) \leq 0$ q.s. By the assumptions (H2) and (H3), it is easy to deduce that $f^{2}-f^{1}+2 G\left(\left(g_{i j}^{2}-g_{i j}^{1}\right)_{i, j=1}^{d}\right) \leq$ 0 q.s.

In the following, we use the notation $\widetilde{\mathbb{E}}_{t}^{l}[\xi]=Y_{t}^{l, \xi}, l=1,2$.

Corollary 16. Let $f^{l}$ and $g_{i j}^{l}$ be deterministic functions and satisfy (H1), (H2), (H3), and (H5) for some $\beta>1, l=1$, 2. If $\widetilde{\mathbb{E}}^{1}[\xi] \geq \widetilde{\mathbb{E}}^{2}[\xi]$ for each $\xi \in L_{G}^{\beta}\left(\Omega_{T}\right)$, then $f^{2}-f^{1}+2 G\left(\left(g_{i j}^{2}-\right.\right.$ $\left.\left.g_{i j}^{1}\right)_{i, j=1}^{d}\right) \leq 0$.

Proof. Taking $\eta_{\varepsilon}$ as in Theorem 15 , since $f^{l}$ and $g_{i j}^{l}$ are deterministic, we could get $\widetilde{\mathbb{E}}_{t}^{l}\left[\eta_{\varepsilon}\right]=\widetilde{\mathbb{E}}^{l}\left[\eta_{\varepsilon}\right]$, for $l=1,2$. And the proof in Theorem 15 still holds true.

4.2. Some Equivalent Relations. We consider the following GBSDE:

$$
\begin{aligned}
Y_{t}= & \xi+\int_{t}^{T} f\left(s, Y_{s}, Z_{s}\right) d s+\int_{t}^{T} g_{i j}\left(s, Y_{s}, Z_{s}\right) d\left\langle B^{i}, B^{j}\right\rangle_{s} \\
& -\int_{t}^{T} Z_{s} d B_{s}-\left(K_{T}-K_{t}\right),
\end{aligned}
$$

where $g_{i j}=g_{j i}$. We use the notation $\widetilde{\mathbb{E}}_{t}[\xi]=Y_{t}$.

Proposition 17. Let $f$ and $g_{i j}$ satisfy (H1), (H2), (H3), (H4), and (H5) for some $\beta>1$ and fix $\alpha \in(1, \beta)$. Then, one has

(1) $\widetilde{\mathbb{E}}_{t}[\xi+\eta]=\widetilde{\mathbb{E}}_{t}[\xi]+\eta$ for $t \in[0, T], \xi \in L_{G}^{\alpha}\left(\Omega_{T}\right)$, and $\eta \in L_{G}^{\alpha}\left(\Omega_{t}\right)$ if and only if for each $t \in[0, T], y, y^{\prime} \in \mathbb{R}$, $z \in \mathbb{R}^{d}$,

$$
\begin{aligned}
& f(t, y, z)-f\left(t, y^{\prime}, z\right) \\
& \quad+2 G\left(\left(g_{i j}(t, y, z)-g_{i j}\left(t, y^{\prime}, z\right)\right)_{i, j=1}^{d}\right)=0 ;
\end{aligned}
$$

(2) $\widetilde{\mathbb{E}}_{t}[\xi+\eta] \leq \widetilde{\mathbb{E}}_{t}[\xi]+\widetilde{\mathbb{E}}_{t}[\eta]$ for $t \in[0, T], \xi \in L_{G}^{\alpha}\left(\Omega_{T}\right)$, and $\eta \in L_{G}^{\alpha}\left(\Omega_{T}\right)$ if and only if for each $t \in[0, T], y$, $y^{\prime} \in \mathbb{R}, z, z^{\prime} \in \mathbb{R}^{d}$,

$$
\begin{gathered}
0 \geq f\left(t, y+y^{\prime}, z+z^{\prime}\right)-f(t, y, z)-f\left(t, y^{\prime}, z^{\prime}\right) \\
+2 G\left(\left(g_{i j}\left(t, y+y^{\prime}, z+z^{\prime}\right)-g_{i j}(t, y, z)\right.\right. \\
\left.\left.-g_{i j}\left(t, y^{\prime}, z^{\prime}\right)\right)_{i, j=1}^{d}\right) ;
\end{gathered}
$$

(3) $\widetilde{\mathbb{E}}_{t}[\lambda \xi+(1-\lambda) \eta] \leq \lambda \widetilde{\mathbb{E}}_{t}[\xi]+(1-\lambda) \widetilde{\mathbb{E}}_{t}[\eta]$ fort $\in[0, T]$, $\lambda \in[0,1], \xi \in L_{G}^{\alpha}\left(\Omega_{T}\right)$, and $\eta \in L_{G}^{\alpha}\left(\Omega_{T}\right)$ if and only if for each $t \in[0, T], y, y^{\prime} \in \mathbb{R}, z, z^{\prime} \in \mathbb{R}^{d}, \lambda \in[0,1]$,

$$
\begin{aligned}
0 \geq f\left(t, \lambda y+(1-\lambda) y^{\prime}, \lambda z+(1-\lambda) z^{\prime}\right) & \\
- & \lambda f(t, y, z)-(1-\lambda) f\left(t, y^{\prime}, z^{\prime}\right) \\
+ & 2 G\left(\left(g_{i j}\left(t, \lambda y+(1-\lambda) y^{\prime}, \lambda z+(1-\lambda) z^{\prime}\right)\right.\right. \\
& \left.\left.-\lambda g_{i j}(t, y, z)-(1-\lambda) g_{i j}\left(t, y^{\prime}, z^{\prime}\right)\right)_{i, j=1}^{d}\right) ;
\end{aligned}
$$

(4) $\widetilde{\mathbb{E}}_{t}[\lambda \xi]=\lambda \widetilde{\mathbb{E}}_{t}[\xi]$ for $t \in[0, T], \lambda \geq 0$, and $\xi \in L_{G}^{\alpha}\left(\Omega_{T}\right)$ if and only if for each $t \in[0, T], y \in \mathbb{R}, z \in \mathbb{R}^{d}, \lambda \geq 0$,

$$
\begin{aligned}
f & (t, \lambda y, \lambda z)-\lambda f(t, y, z) \\
& =2 G\left(\left(\lambda g_{i j}(t, y, z)-g_{i j}(t, \lambda y, \lambda z)\right)_{i, j=1}^{d}\right) \\
& =-2 G\left(\left(g_{i j}(t, \lambda y, \lambda z)-\lambda g_{i j}(t, y, z)\right)_{i, j=1}^{d}\right) .
\end{aligned}
$$

Proof. (1) “ $\Rightarrow$ " part. For each fixed $t \in[0, T), y, y^{\prime} \in \mathbb{R}, z \in$ $\mathbb{R}^{d}$, we take

$$
\begin{aligned}
\xi_{\varepsilon}= & y+\left\langle z, h_{i j}\right\rangle\left(\left\langle B^{i}, B^{j}\right\rangle_{t+\varepsilon}-\left\langle B^{i}, B^{j}\right\rangle_{t}\right) \\
& +\left\langle z, B_{t+\varepsilon}-B_{t}\right\rangle, \quad \eta=y^{\prime}-y,
\end{aligned}
$$

where $h_{i j}=h_{j i} \in \mathbb{R}^{d}$. Then, by Theorem 12 and $\widetilde{\mathbb{E}}_{t}\left[\xi_{\varepsilon}+\eta\right]=$ $\widetilde{\mathbb{E}}_{t}\left[\xi_{\varepsilon}\right]+\eta$, we can obtain

$$
\begin{aligned}
& f\left(t, y^{\prime}, z\right)+2 G\left(\left(g_{i j}\left(t, y^{\prime}, z\right)+\left\langle z, h_{i j}\right\rangle\right)_{i, j=1}^{d}\right) \\
& \quad=f(t, y, z)+2 G\left(\left(g_{i j}(t, y, z)+\left\langle z, h_{i j}\right\rangle\right)_{i, j=1}^{d}\right) .
\end{aligned}
$$

We choose $h_{i j}$ such that $g_{i j}\left(t, y^{\prime}, z\right)+\left\langle z, h_{i j}\right\rangle=0$, which implies (47).

“ $\Leftarrow$ " part. Let $(Y, Z, K)$ be the solution of $G$-BSDE (46) corresponding to terminal condition $\xi$. We claim that $\left(Y_{s}+\right.$ $\left.\eta, Z_{s}, K_{s}\right)_{s \in[t, T]}$ is the solution of G-BSDE (46) corresponding 
to terminal condition $\xi+\eta$ on $[t, T]$. For this, we only need to check that, for $s \in[t, T]$,

$$
\begin{array}{rl}
\int_{s}^{T} & f\left(u, Y_{u}, Z_{u}\right) d u+\int_{s}^{T} g_{i j}\left(u, Y_{u}, Z_{u}\right) d\left\langle B^{i}, B^{j}\right\rangle_{u} \\
= & \int_{s}^{T} f\left(u, Y_{u}+\eta, Z_{u}\right) d u \\
& +\int_{s}^{T} g_{i j}\left(u, Y_{u}+\eta, Z_{u}\right) d\left\langle B^{i}, B^{j}\right\rangle_{u} .
\end{array}
$$

By (47) we can get

$$
\begin{aligned}
& \int_{s}^{T}\left(g_{i j}\left(u, Y_{u}, Z_{u}\right)-g_{i j}\left(u, Y_{u}+\eta, Z_{u}\right)\right) d\left\langle B^{i}, B^{j}\right\rangle_{u} \\
& \quad-2 \int_{s}^{T} G\left(\left(g_{i j}\left(u, Y_{u}, Z_{u}\right)-g_{i j}\left(u, Y_{u}+\eta, Z_{u}\right)\right)_{i, j=1}^{d}\right) d u \\
& =\int_{s}^{T}\left(g_{i j}\left(u, Y_{u}, Z_{u}\right)-g_{i j}\left(u, Y_{u}+\eta, Z_{u}\right)\right) d\left\langle B^{i}, B^{j}\right\rangle_{u} \\
& \quad+\int_{s}^{T}\left(f\left(u, Y_{u}, Z_{u}\right)-f\left(u, Y_{u}+\eta, Z_{u}\right)\right) d u \leq 0, \\
& \int_{s}^{T} \quad\left(g_{i j}\left(u, Y_{u}+\eta, Z_{u}\right)-g_{i j}\left(u, Y_{u}, Z_{u}\right)\right) d\left\langle B^{i}, B^{j}\right\rangle_{u} \\
& \quad-2 \int_{s}^{T} G\left(\left(g_{i j}\left(u, Y_{u}+\eta, Z_{u}\right)-g_{i j}\left(u, Y_{u}, Z_{u}\right)\right)_{i, j=1}^{d}\right) d u \\
& =\int_{s}^{T}\left(g_{i j}\left(u, Y_{u}+\eta, Z_{u}\right)-g_{i j}\left(u, Y_{u}, Z_{u}\right)\right) d\left\langle B^{i}, B^{j}\right\rangle_{u} \\
& \quad+\int_{s}^{T}\left(f\left(u, Y_{u}+\eta, Z_{u}\right)-f\left(u, Y_{u}, Z_{u}\right)\right) d u \leq 0,
\end{aligned}
$$

which implies (53). The proof of (1) is complete.

(2) “ $\Rightarrow$ ” part. For each fixed $t \in[0, T), y, y^{\prime} \in \mathbb{R}, z, z^{\prime} \in$ $\mathbb{R}^{d}$, we consider $\xi_{\varepsilon}=y+\left\langle z, h_{i j}\right\rangle\left(\left\langle B^{i}, B^{j}\right\rangle_{t+\varepsilon}-\left\langle B^{i}, B^{j}\right\rangle_{t}\right)+$ $\left\langle z, B_{t+\varepsilon}-B_{t}\right\rangle$ and $\eta_{\varepsilon}=y^{\prime}+\left\langle z^{\prime}, h_{i j}^{\prime}\right\rangle\left(\left\langle B^{i}, B^{j}\right\rangle_{t+\varepsilon}-\left\langle B^{i}, B^{j}\right\rangle_{t}\right)+$ $\left\langle z^{\prime}, B_{t+\varepsilon}-B_{t}\right\rangle$, where $h_{i j}=h_{j i} \in \mathbb{R}^{d}$ and $h_{i j}^{\prime}=h_{j i}^{\prime} \in \mathbb{R}^{d}$. Then, by Theorem 12 and $\widetilde{\mathbb{E}}_{t}\left[\xi_{\varepsilon}+\eta_{\varepsilon}\right]=\widetilde{\mathbb{E}}_{t}\left[\xi_{\varepsilon}\right]+\widetilde{\mathbb{E}}_{t}\left[\eta_{\varepsilon}\right]$, we obtain

$$
\begin{gathered}
f\left(t, y+y^{\prime}, z+z^{\prime}\right) \\
+2 G\left(\left(g_{i j}\left(t, y+y^{\prime}, z+z^{\prime}\right)\right.\right. \\
\left.\left.+\left\langle z, h_{i j}\right\rangle+\left\langle z^{\prime}, h_{i j}^{\prime}\right\rangle\right)_{i, j=1}^{d}\right) \\
\leq f(t, y, z)+f\left(t, y^{\prime}, z^{\prime}\right) \\
+2 G\left(\left(g_{i j}(t, y, z)+\left\langle z, h_{i j}\right\rangle\right)_{i, j=1}^{d}\right) \\
+2 G\left(\left(g_{i j}\left(t, y^{\prime}, z^{\prime}\right)+\left\langle z^{\prime}, h_{i j}^{\prime}\right\rangle\right)_{i, j=1}^{d}\right) .
\end{gathered}
$$

We choose $h_{i j}, h_{i j}^{\prime}$ such that $g_{i j}(t, y, z)+\left\langle z, h_{i j}\right\rangle=0$ and $g_{i j}\left(t, y^{\prime}, z^{\prime}\right)+\left\langle z^{\prime}, h_{i j}^{\prime}\right\rangle=0$, which implies (48).

" $\Leftarrow$ " part. Let $(Y, Z, K)$ and $\left(Y^{\prime}, Z^{\prime}, K^{\prime}\right)$ be the solutions of $G$-BSDE (46) corresponding to terminal condition $\xi$ and $\eta$, respectively. Then, $\left(Y+Y^{\prime}, Z+Z^{\prime}, K\right)$ solves the following G-BSDE:

$$
\begin{aligned}
Y_{t}+Y_{t}^{\prime}= & \xi+\eta+\int_{t}^{T} f\left(s, Y_{s}+Y_{s}^{\prime}, Z_{s}+Z_{s}^{\prime}\right) d s \\
& +\int_{t}^{T} g_{i j}\left(s, Y_{s}+Y_{s}^{\prime}, Z_{s}+Z_{s}^{\prime}\right) d\left\langle B^{i}, B^{j}\right\rangle_{s} \\
& +V_{T}-V_{t}-\int_{t}^{T}\left(Z_{s}+Z_{s}^{\prime}\right) d B_{s}-\left(K_{T}-K_{t}\right),
\end{aligned}
$$

where

$$
\begin{aligned}
& V_{t}=-K_{t}^{\prime}-\int_{0}^{t}\left(f\left(s, Y_{s}+Y_{s}^{\prime}, Z_{s}+Z_{s}^{\prime}\right)\right. \\
& \left.-f\left(s, Y_{s}, Z_{s}\right)-f\left(s, Y_{s}^{\prime}, Z_{s}^{\prime}\right)\right) d s \\
& -\int_{0}^{t}\left(g_{i j}\left(s, Y_{s}+Y_{s}^{\prime}, Z_{s}+Z_{s}^{\prime}\right)-g_{i j}\left(s, Y_{s}, Z_{s}\right)\right. \\
& \left.-g_{i j}\left(s, Y_{s}^{\prime}, Z_{s}^{\prime}\right)\right) d\left\langle B^{i}, B^{j}\right\rangle_{s} \\
& =-K_{t}^{\prime}-\left\{\int _ { 0 } ^ { t } \left(g_{i j}\left(s, Y_{s}+Y_{s}^{\prime}, Z_{s}+Z_{s}^{\prime}\right)\right.\right. \\
& \left.-g_{i j}\left(s, Y_{s}, Z_{s}\right)-g_{i j}\left(s, Y_{s}^{\prime}, Z_{s}^{\prime}\right)\right) d\left\langle B^{i}, B^{j}\right\rangle_{s} \\
& -2 \int_{0}^{t} G\left(\left(g_{i j}\left(s, Y_{s}+Y_{s}^{\prime}, Z_{s}+Z_{s}^{\prime}\right)\right.\right. \\
& -g_{i j}\left(s, Y_{s}, Z_{s}\right) \\
& \left.\left.\left.-g_{i j}\left(s, Y_{s}^{\prime}, Z_{s}^{\prime}\right)\right)_{i, j=1}^{d}\right) d s\right\} \\
& -\int_{0}^{t}\left\{f\left(s, Y_{s}+Y_{s}^{\prime}, Z_{s}+Z_{s}^{\prime}\right)\right. \\
& -f\left(s, Y_{s}, Z_{s}\right)-f\left(s, Y_{s}^{\prime}, Z_{s}^{\prime}\right) \\
& +2 G\left(\left(g_{i j}\left(s, Y_{s}+Y_{s}^{\prime}, Z_{s}+Z_{s}^{\prime}\right)-g_{i j}\left(s, Y_{s}, Z_{s}\right)\right.\right. \\
& \left.\left.\left.-g_{i j}\left(s, Y_{s}^{\prime}, Z_{s}^{\prime}\right)\right)_{i, j=1}^{d}\right)\right\} d s .
\end{aligned}
$$

By (48), it is easy to check that $V_{t}$ is an increasing process. Then, by Theorem 11 , we can get $\widetilde{\mathbb{E}}_{t}[\xi+\eta] \leq \widetilde{\mathbb{E}}_{t}[\xi]+\widetilde{\mathbb{E}}_{t}[\eta]$. The proof of (2) is complete.

Finally, we could prove (3) as in (2) and (4) as in (1).

Proposition 18. One has the following.

(1) If $G(A)+G(-A)>0$ for any $A \in \mathbb{S}_{d}$ and $A \neq 0$, then (47) holds if and only if $f$ and $g_{i j}$ are independent of $y$. 
(2) If there exists an $A \in \mathbb{S}_{d}$ with $A \neq 0$ such that $G(A)+G(-A)=0$ and $G(A) \neq 0$, then, for any fixed $g(t, y, z)$ satisfying $(H 1)-(H 5)$, one has $f(t, y, z)=$ $-2 G(A) g(t, y, z)$ and $\left(g_{i j}(t, y, z)\right)_{i, j=1}^{d}=g(t, y, z) A$ satisfying (47).

Proof. It is easy to verify (2), and we only need to prove (1). If (47) holds, it is easy to check that $G\left(\left(g_{i j}(t, y, z)-\right.\right.$ $\left.\left.g_{i j}(t, 0, z)\right)_{i, j=1}^{d}\right)+G\left(\left(g_{i j}(t, 0, z)-g_{i j}(t, y, z)\right)_{i, j=1}^{d}\right)=0$ holds. Then, from the assumption, we get $g_{i j}(t, y, z)=g_{i j}(t, 0, z)$. Therefore, by (47), we have $f(t, y, z)=f(t, 0, z)$, which implies that $f$ and $g_{i j}$ are independent of $y$. The converse part is obvious.

\section{Acknowledgments}

K. He acknowledges the financial support from the National Natural Science Foundation of China (Grant nos. 11301068 and 11171062) and the Innovation Program of Shanghai Municipal Education Commission (Grant no. 12ZZ063). M. $\mathrm{Hu}$ acknowledges the financial support from the National Natural Science Foundation of China (Grant nos. 11201262 and 11101242) and the Scientific Research Foundation for the Excellent Middle-Aged and Young Scientists of Shandong Province of China (Grant no. BS2013SF020).

\section{References}

[1] É. Pardoux and S. G. Peng, "Adapted solution of a backward stochastic differential equation," Systems \& Control Letters, vol. 14, no. 1, pp. 55-61, 1990.

[2] S. Peng, "Backward SDE and related g-expectation," in Backward Stochastic Differential Equations, N. El Karoui and L. Mazliak, Eds., vol. 364 of Pitman Research Notes in Mathematics Series, pp. 141-159, 1997.

[3] Z. J. Chen, "A property of backward stochastic differential equations," Comptes Rendus de l'Académie des Sciences. Série I, vol. 326, no. 4, pp. 483-488, 1998.

[4] P. Briand, F. Coquet, Y. Hu, J. Mémin, and S. G. Peng, "A converse comparison theorem for BSDEs and related properties of $g$-expectation," Electronic Communications in Probability, vol. 5, pp. 101-117, 2000.

[5] L. Jiang, "Representation theorems for generators of backward stochastic differential equations," Comptes Rendus Mathématique. Académie des Sciences. Paris I, vol. 340, no. 2, pp. 161-166, 2005.

[6] L. Jiang, "Converse comparison theorems for backward stochastic differential equations," Statistics \& Probability Letters, vol. 71, no. 2, pp. 173-183, 2005.

[7] L. Jiang, "Convexity, translation invariance and subadditivity for $g$-expectations and related risk measures," The Annals of Applied Probability, vol. 18, no. 1, pp. 245-258, 2008.

[8] S. Peng, "Filtration consistent nonlinear expectations and evaluations of contingent claims," Acta Mathematicae Applicatae Sinica, vol. 20, no. 2, pp. 191-214, 2004.

[9] S. Peng, "Nonlinear expectations and nonlinear Markov chains," Chinese Annals of Mathematics B, vol. 26, no. 2, pp. 159-184, 2005.
[10] S. Peng, "G-expectation, G-Brownian motion and related stochastic calculus of Itô type," in Stochastic Analysis and Applications, vol. 2 of The Abel Symposium, pp. 541-567, Springer, Berlin, Germany, 2007.

[11] S. Peng, "G-Brownian motion and dynamic risk measureunder volatility uncertainty," http://arxiv.org/abs/0711.2834v1.

[12] S. Peng, "Multi-dimensional G-Brownian motion and related stochastic calculus under G-expectation," Stochastic Processes and Their Applications, vol. 118, no. 12, pp. 2223-2253, 2008.

[13] S. Peng, "A new central limit theorem under sublinear expectations," http://arxiv.org/abs/0803.2656v1.

[14] H. M. Soner, N. Touzi, and J. Zhang, "Wellposedness of second order backward SDEs," Probability Theory and Related Fields, vol. 153, no. 1-2, pp. 149-190, 2012.

[15] M. S. Hu, S. L. Ji, S. G. Peng, and Y. S. Song, "Backward stochastic differential equations driven by G-brownian motion," http://arxiv.org/abs/1206.5889v1.

[16] M. S. Hu, S. L. Ji, S. G. Peng, and Y. S. Song, "Comparison theorem, Feynman-Kac formula and Girsanov transformation for BSDEs driven by G-Brownian motion," http://arxiv.org/abs/ 1212.5403vl.

[17] S. Peng, "G.G-Brownian motion and dynamic risk measure under volatility uncertainty," http://arxiv.org/abs/0711.2834v1.

[18] S. G. Peng, "Multi-dimensional G-Brownian motion and related stochastic calculus under G-expectation," Stochastic Processes and their Applications, vol. 118, no. 12, pp. 2223-2253, 2008.

[19] S. G. Peng, "Nonlinear expectations and stochastic calculus under uncertainty," http://arxiv.org/abs/1002.4546vl.

[20] Y. S. Song, "Some properties on G-evaluation and its applications to G-martingale decomposition," Science China Mathematics, vol. 54, no. 2, pp. 287-300, 2011. 


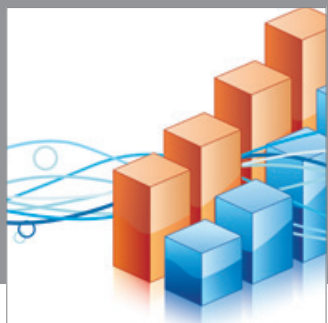

Advances in

Operations Research

mansans

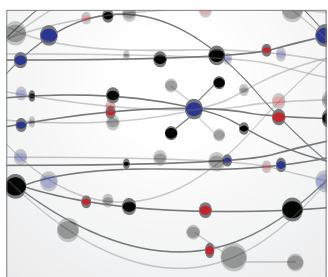

The Scientific World Journal
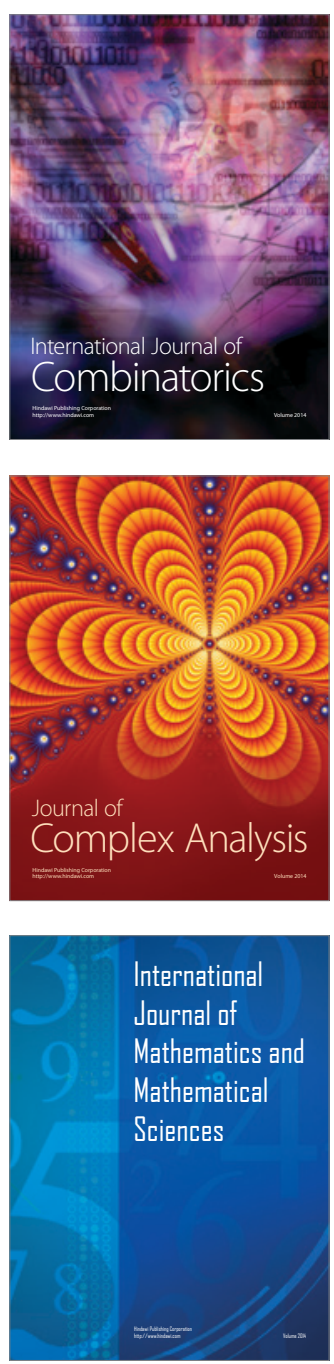
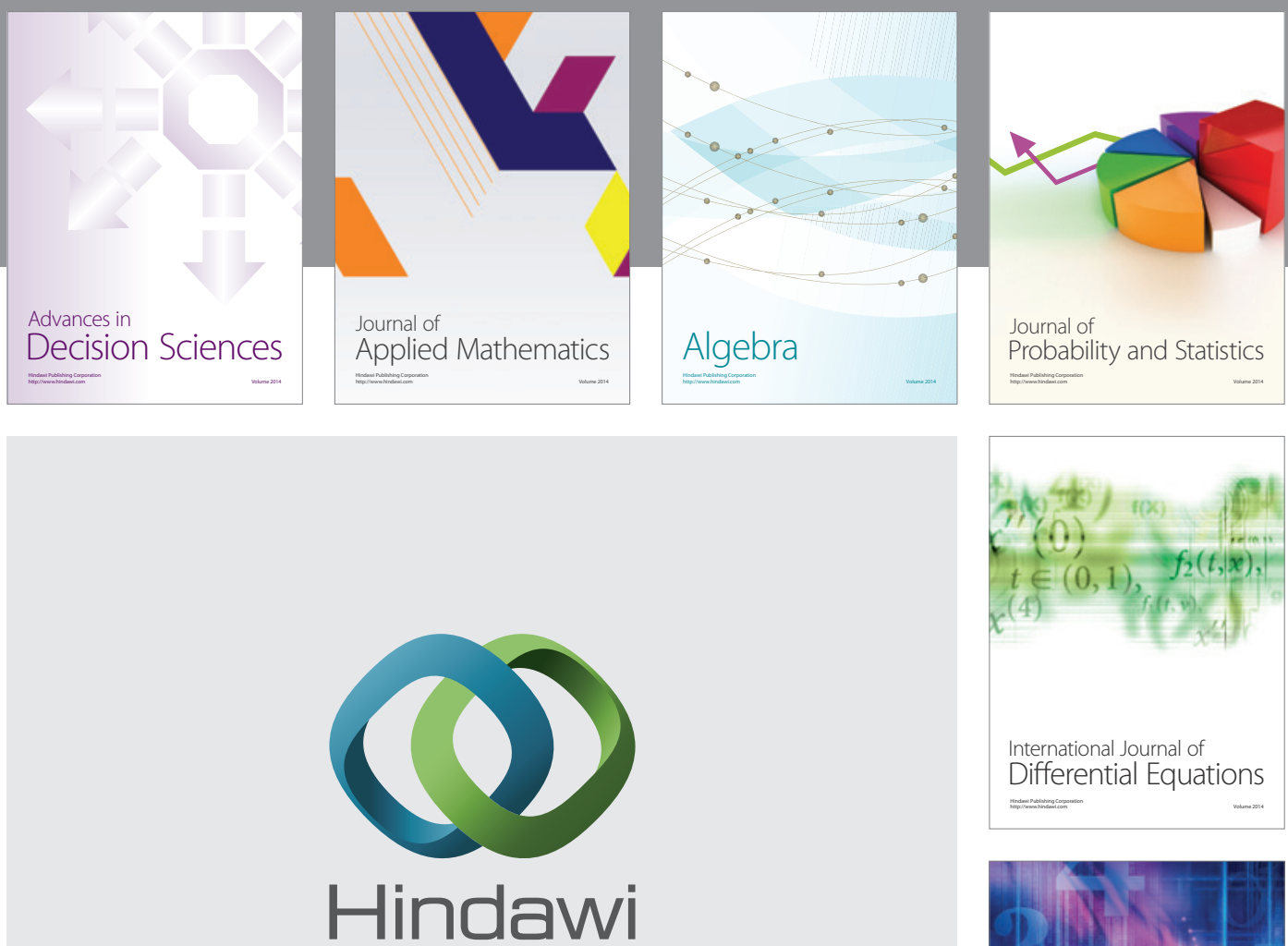

Submit your manuscripts at http://www.hindawi.com
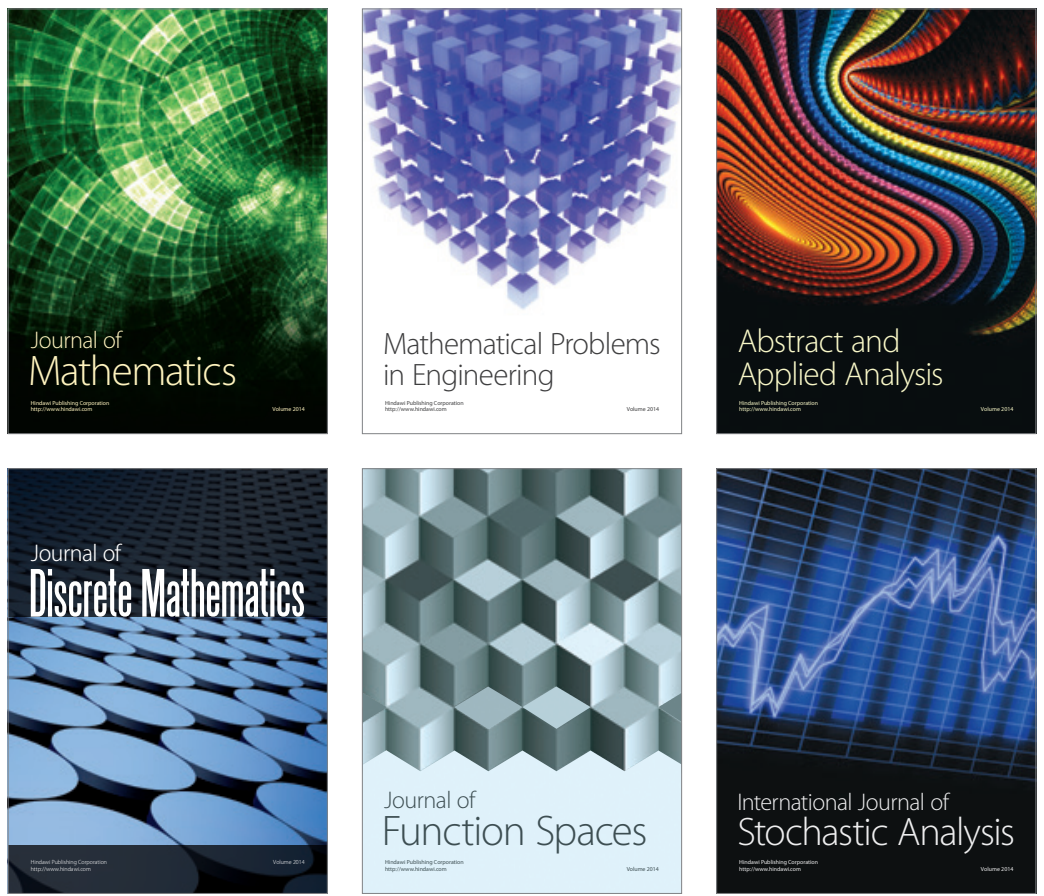

Journal of

Function Spaces

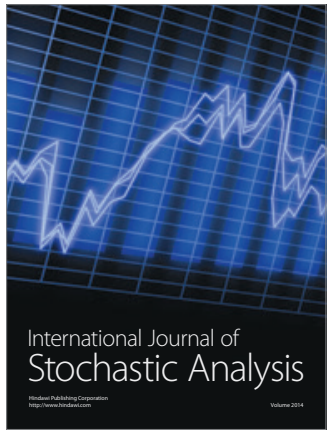

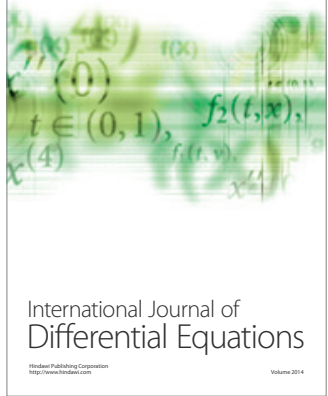
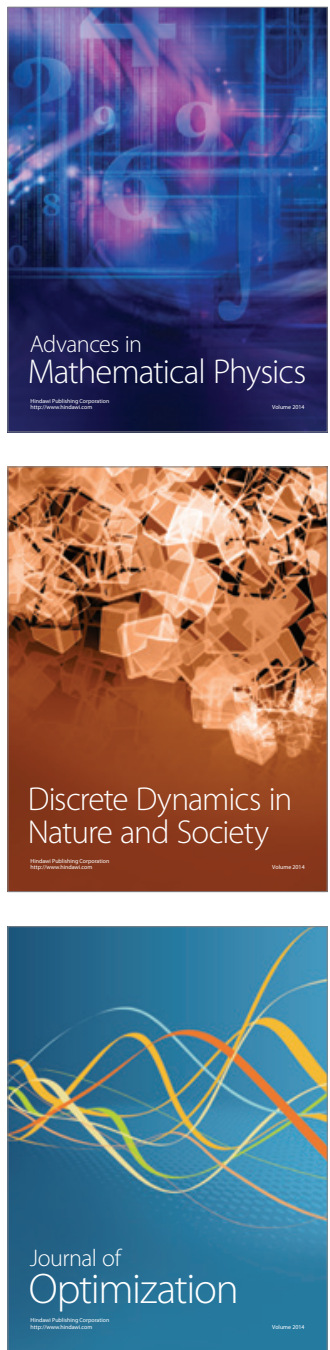